\title{
Miodrag Mićić (Ed): Sample Preparation Techniques for Soil, Plant and Animal Samples
}

\author{
Lourde $\operatorname{Ramos}^{1}$
}

(C) Springer-Verlag Berlin Heidelberg 2016

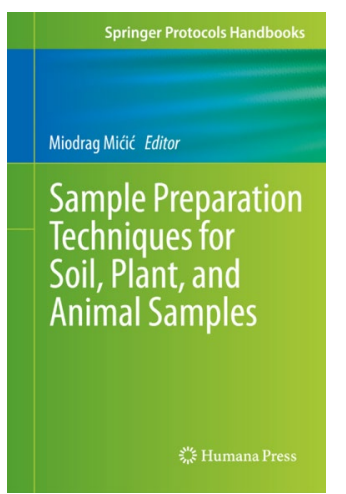

\section{Bibliography}

Sample Preparation Techniques for Soil, Plant and Animal Samples

Miodrag Mićić (Ed)

Series: Springer Protocols Handbooks Humana Press, Heidelberg, Germany, 2016, pp 406

ISBN 978-1-4939-3184-2

EUR 101.64, USD 119.00

Almost irrespective of the application area, sample preparation is usually recognised as the bottleneck of most modern analytical procedures. In general, the several steps involved in sample treatment take advantage of particular physicochemical properties of the analytes of interest to isolate them from other matrix components without affecting their nature. However, for the many analyte-matrix combinations or, more precisely, the many anticipated and unanticipated analyte-interference mixtures, the approach to be applied for efficient extraction plus purification of the target analytes is not always obvious. As a consequence, in most application fields, a variety of sample treatment protocols have been reported in the literature for essentially similar applications and there is a general lack of texts summarising the basic knowledge regarding sample preparation for each specific research area.

Lourde Ramos

1.ramos@iqog.csic.es

1 Department Instrumental Analysis \& Environmental Chemistry, IQOG-CSIC, Juan de la Cierva 3, 28006 Madrid, Spain
This book summarises the theoretical and practical aspects of sample preparation for molecular biology, proteomics and glycomics-related studies in a single and, therefore, quite unique text. The monograph is organised in 28 succinct and to-the-point chapters dealing with quite diverse topics within the three main thematic blocks. In the first section of the book, the authors describe the physical and chemical properties of nucleic acids (Chapter 1), introduce basic statistical concepts and sampling techniques and methods (Chapters 3 and 4) and, in particular, discuss in detail the main aspects related to sample preparation of nucleic acids and proteins in difficult samples. In this text, difficult samples are defined as those complicated by lyse or especially complex, i.e., with many potential interfering compounds, such as food, feed and semi-solid environmental matrices. Techniques and approaches for the purification and isolation of nucleic acids from complex matrices, including refractory material, are summarised in Chapter 4; while the benefits of using magnetic beads over other conventional nucleic purification techniques such as membrane separation or resins are presented in Chapter 5. Chapters 6-9 review and discus the respective merits and limitations of the different mechanical technologies available for sample lysis, grinding and homogenisation for molecular biology applications. Among them, special attention has been paid to the beadbeating technology. The description of the most relevant bench-top commercial systems, the parameters to consider during method optimisation, and the benefices of its application to the homogenisation of samples difficult to lyse are summarised in Chapters 7 and 8 and discussed through a number of representation application studies. Portable systems are described in detail in Chapter 9, where an indication of their respective application ranges is also provided.

The second thematic block of the book (Chapters 10-25) consists of a succession of study cases dealing with the 
determination of nucleic acids in a variety of complex and difficult-to-analyse samples. Matrices considered range from food to GMO, seeds and yeasts, and from stem cells to mammalian and environmental samples. In all cases, relevant general considerations and theoretical aspects are discussed, and stepwise protocols and practical tips provided. Present limitations, remaining challenges and desirable future developments are also identified.

The last part of the book includes three chapters that focus on the extraction and purification of other relevant macromolecules, viz., lignin and cellulose (Chapter 26), glycans (Chapter 27) and prions (Chapter 28).
Written as a multi-authored text by pioneers and specialist in their respective research fields, both from academia and industry, with a dynamic and concise style, this monograph summarises and presents in an organised single text scattered relevant background information concerning sample preparation for a variety of molecular biology analyses, together with a number of protocols for successful treatment of difficult matrices. This book can be considered a useful practical guide for all those involved in sample preparation within the molecular biology practice, but it is also suitable for newcomers trying to obtain a general, but complete overview of the subject. 